\title{
Tentative Set of Key Values for Thermodynamics-Part I. Report of the ICSU-CODATA Task Group on Key Values for Thermodynamics, October, 1970*
}

\author{
S. Sunner**
}

(December 9, 1970)

\begin{abstract}
This paper is a preliminary report from the Task Group on Key Values for Thermodynamics of the Committee on Data for Science and Technology (CODATA) of the International Council of Scientific Unions (ICSU). It gives values agreed upon by the Committee for enthalpies of formation and entropies of 32 atoms, molecules, and ionic species. Sources of the data are also tabulated.

Key words: CODATA; key values; thermodynamics.

EDITOR's NOTE: In line with the Bureau's role in support of international scientific collaboration efforts of the International Council of Scientific Unions (ICSU), the Journal of Research is publishing this preliminary report on Key Values for Thermodynamies, as prepared by a Task Group of the Committee on Data for Science and Technology (CODATA). It is hoped that the widespread distribution of this report will provide an opportunity for all interested scientists to comment on the proposed values and thus aid the members of the Task Group in their final evaluations. The members of the Task Group are: S. Sunner (Sweden), Chairman, J. D. Cox (U.K.), L. V. Gurvich (U.S.S.R.), D. M. Newitt (U.K.), D. D. Wagman (U.S.A.); consultants to the Group are: C. B. Alcock (Canada), W. H. Evans (U.S.A.), V. A. Medvedev (U.S.S.R.), and I. Wadsö (Sweden). This report has appeared as CODATA Bulletin No. 2, November, 1970.
\end{abstract}

In 1968, the Committee on Data for Science and Technology (CODATA) of the International Council of Scientific Unions (ICSU) appointed a Task Group on Key Values for Thermodynamics. The first objective of the Task Group is to prepare an internationally agreed set of values of the basic thermodynamic properties of a number of chemical species, including the elements in both standard and monatomic gaseous states, aqueous ions, and simple compounds.

The table below contains the Task Group's first set of proposed values for the standard enthalpies of formation at $298.15 \mathrm{~K}, \Delta H_{f, 298.15}^{\circ}$, the standard entropies at $298.15 \mathrm{~K}, S_{298.15}^{\circ}$, and the standard enthalpy increments between $0 \mathrm{~K}$ and $298.15 \mathrm{~K}, H_{298.15}^{\circ}-H_{0}^{\circ}$, of 32 species. The values are based on selections and calculations made at the National Bureau of Standards, U.S.A., and the Institute for High Temperatures of the Academy of Sciences of U.S.S.R., and agreed by the whole Task Group.

By this announcement, the Task Group invites comments from thermodynamicists on the aptness of the proposed values. Any such comments will be gladly received by the Chairman of the Task Group, and will help in the formulation of a first set of recommended key values, to be published in approximately one year's time. Until then, any author who wishes to cite a value

*An invited paper

** Chairman, CODATA Task Group on Key Values for Thermodynamics. Thermochemistry Laboratory, Lund University, POB 740, S-220 07, Lund 7, Sweden. from the table below is requested to make clear that the value he cites is tentative. It should be carefully noted that the values now proposed are not fully consistent with the values in any previously published thermodynamic tables, for example the "Technical Notes 270" of the National Bureau of Standards or "Termicheskie Konstanty Weshchesty" of the Academy of Sciences of U.S.S.R. The achievement of consistency between the various thermodynamic tables is of course a long-term objective which the Task Group hopes to facilitate, but for some time to come, inconsistency between the Task Group's recommended values and values already tabulated in other series is inevitable.

The second set of species planned for consideration by the Task Group in the immediate future is likely to include the following: $\mathrm{S}(\mathrm{g}), \mathrm{S}(\mathrm{rh}), \mathrm{SO}_{2}(\mathrm{~g}), \mathrm{SO}_{4}^{2-}(\mathrm{aq})$, $\mathrm{NH}_{3}(\mathrm{~g}), \mathrm{NH}_{4}^{+}(\mathrm{aq}), \mathrm{NO}_{3}^{-}(\mathrm{aq}), \mathrm{OH}^{-}(\mathrm{aq}), \mathrm{Na}(\mathrm{g}), \mathrm{Na}(\mathrm{c})$, $\mathrm{Na}^{+}(\mathrm{aq}), \mathrm{K}(\mathrm{g}), \mathrm{K}(\mathrm{c}), \mathrm{K}^{+}(\mathrm{aq}), \mathrm{Li}(\mathrm{g}), \mathrm{Li}(\mathrm{c}), \mathrm{Li}^{+}(\mathrm{aq}), \mathrm{Rb}(\mathrm{g})$, $\mathrm{Rb}(\mathrm{c}), \mathrm{Rb}^{+}(\mathrm{aq}), \mathrm{Cs}(\mathrm{g}), \mathrm{Cs}(\mathrm{c}), \mathrm{Cs}^{+}(\mathrm{aq}), \mathrm{F}(\mathrm{g}), \mathrm{F}_{2}(\mathrm{~g})$, $\mathrm{HF}(\mathrm{g}), \mathrm{F}^{-}(\mathrm{aq})$.

It would assist the Task Group if researchers who have completed measurements that are relevant to the Task Group's work on the second set of species would communicate their results to the Chairman of the Task Group in advance of publication.

In the table below, the usual definitions of standard states have been adopted. For crystalline solids (symbolized as c) and for liquids (symbolized as l), the standard state is that of the pure substance under 
a pressure of $101325 \mathrm{~Pa}$ (formerly known as 1 standard atmosphere). For gases (symbolized as $g$ ), the standard state is that of the ideal gas at a pressure of $101325 \mathrm{~Pa}$. For aqueous solutions (symbolized as aq), the standard state is the hypothetical ideal solution at a concentration of $1 \mathrm{~mol} \mathrm{~kg}^{-1}$ (of solvent); the properties of ideal aqueous ionic solutions are taken equal to the sum of the properties of the individual ions.

The following values of the fundamental constants were employed in the calculations: gas constant,
$R,(8.31433 \pm 0.00120) \mathrm{J} \mathrm{mol}^{-1} \mathrm{~K}^{-1}$; constant relating wave number and energy $(0.1196256 \pm 0.0000107)$ $\mathrm{J} \mathrm{m} \mathrm{mol}{ }^{-1}$. Relative atomic masses were taken as recommended by the Commission on Atomic Weights of IUPAC (1970). All thermodynamic data in the table relate to the natural mixture of isotopic species. Nuclear spin contributions were ignored. The uncertainties in the table represent the Task Group's estimates of the overall uncertainties at a 95 percent confidence level.

Tentative Set of Key Values for Thermodynamics - Part I

\begin{tabular}{|c|c|c|c|c|c|c|}
\hline Substance & State & $\begin{array}{l}\Delta H^{\circ}, 298.15 \\
\mathrm{~kJ} \mathrm{~mol}^{-1}\end{array}$ & $\begin{array}{l}\text { Foot- } \\
\text { note }\end{array}$ & $\underset{\mathrm{J} \mathrm{mol}^{-1} \mathrm{~K}^{-1}}{S_{298.15}^{\circ}}$ & $\begin{array}{c}H^{\circ}{ }_{298.15}-H^{\circ}{ }_{0} \\
\mathrm{~kJ} \mathrm{~mol}^{-1}\end{array}$ & $\begin{array}{l}\text { Foot- } \\
\text { note }\end{array}$ \\
\hline $\mathrm{O}$ & $g$ & $249.17 \pm 0.10$ & c & $160.946 \pm 0.020$ & $6.728 \pm 0.003$ & $\mathrm{~b}$ \\
\hline $\mathrm{O}_{2}$ & $\mathrm{~g}$ & 0 & a & $205.037 \pm 0.033$ & $8.682 \pm 0.004$ & $\mathrm{~b}$ \\
\hline $\mathrm{H}^{-}$ & $\mathrm{g}$ & $217.997 \pm 0.006 \dagger$ & d & $114.604 \pm 0.015$ & $6.197 \pm 0.002$ & $\mathrm{~b}$ \\
\hline $\mathrm{H}^{+}$ & $\mathrm{aq}$ & 0 & a & 0 & - & a \\
\hline $\mathrm{H}_{2}$ & $\mathrm{~g}$ & 0 & a & $130.570 \pm 0.033$ & $8.468 \pm 0.003$ & $\mathrm{~b}$ \\
\hline $\mathrm{H}_{2} \mathrm{O}$ & l & $-285.830 \pm 0.042$ & $\mathrm{e}$ & $69.950 \pm 0.080$ & - & $\mathrm{f}$ \\
\hline $\mathrm{H}_{2} \mathrm{O}$ & $\mathrm{g}$ & $-241.814 \pm 0.042$ & $g$ & $188.724 \pm 0.040$ & $9.908 \pm 0.008$ & $\mathrm{~b}$ \\
\hline $\mathrm{He}$ & $\mathrm{g}$ & 0 & $\mathrm{a}$ & $126.039 \pm 0.012$ & $6.197 \pm 0.002$ & $\mathrm{~b}$ \\
\hline $\mathrm{Ne}$ & $\mathrm{g}$ & 0 & $\mathrm{a}$ & $146.214 \pm 0.016$ & $6.197 \pm 0.002$ & $\mathrm{~b}$ \\
\hline $\mathrm{Ar}$ & $\mathrm{g}$ & 0 & $\mathrm{a}$ & $154.732 \pm 0.020$ & $6.197 \pm 0.002$ & $\mathrm{~b}$ \\
\hline $\mathrm{Kr}$ & g & 0 & a & $163.971 \pm 0.020$ & $6.197 \pm 0.002$ & b \\
\hline $\mathrm{Xe}$ & $\mathrm{g}$ & 0 & a & $169.573 \pm 0.020$ & $6.197 \pm 0.002$ & $\mathrm{~b}$ \\
\hline $\mathrm{Cl}$ & $\mathrm{g}$ & $121.290 \pm 0.008$ & $\mathrm{~h}$ & $165.076 \pm 0.020$ & $6.272 \pm 0.003$ & $\mathrm{~b}$ \\
\hline $\mathrm{Cl}^{-}$ & $\mathrm{aq}$ & $-167.08 \pm 0.14$ & $\mathrm{i}$ & $56.78 \pm 0.60$ & - & i \\
\hline $\mathrm{Cl}_{2}$ & $g$ & 0 & a & $222.965 \pm 0.040$ & $9.180 \pm 0.008$ & $\mathrm{~b}$ \\
\hline $\mathrm{HCl}$ & $\mathrm{g}$ & $-92.31 \pm 0.13$ & $\mathrm{i}$ & $186.786 \pm 0.033$ & $8.640 \pm 0.004$ & $\mathrm{~b}$ \\
\hline $\mathrm{Br}$ & $\mathrm{g}$ & $111.84 \pm 0.12$ & $\mathrm{j}$ & $174.904 \pm 0.020$ & $6.197 \pm 0.002$ & $\mathrm{~b}$ \\
\hline $\mathrm{Br}^{-}$ & $\mathrm{aq}$ & $-121.50 \pm 0.17$ & $\mathrm{k}$ & $83.3 \pm 1.3$ & - & $\mathrm{k}$ \\
\hline $\mathrm{Br}_{2}$ & l & 0 & a & $152.210 \pm 0.040$ & $24.52 \pm 0.13$ & l \\
\hline $\mathrm{Br}_{2}$ & $\mathrm{~g}$ & $30.91 \pm 0.11$ & 1 & $245.350 \pm 0.054$ & $9.724 \pm 0.012$ & b \\
\hline $\mathrm{HBr}$ & $g$ & $-36.38 \pm 0.17$ & $\mathrm{k}$ & $198.585 \pm 0.033$ & $8.648 \pm 0.004$ & $\mathrm{~b}$ \\
\hline I & $\mathrm{g}$ & $106.762 \pm 0.040$ & $\mathrm{~m}$ & $180.673 \pm 0.020$ & $6.197 \pm 0.002$ & b \\
\hline $\mathrm{I}^{-}$ & aq & $-56.90 \pm 0.84$ & $\mathrm{n}$ & $107.11 \pm 0.42$ & - & $\mathrm{n}$ \\
\hline $\mathrm{I}_{2}$ & c & 0 & a & $116.139 \pm 0.080$ & $13.196 \pm 0.040$ & o \\
\hline $\mathrm{I}_{2}$ & $g$ & $62.421 \pm 0.080$ & o & $260.567 \pm 0.063$ & $10.117 \pm 0.012$ & $\mathrm{~b}$ \\
\hline HI & $\mathrm{g}$ & $26.36 \pm 0.80$ & $\mathrm{n}$ & $206.480 \pm 0.040$ & $8.657 \pm 0.006$ & $\mathrm{~b}$ \\
\hline $\mathrm{N}$ & $\mathrm{g}$ & $472.68 \pm 0.40$ & $\mathrm{p}$ & $153.189 \pm 0.020$ & $6.197 \pm 0.002$ & b \\
\hline $\mathrm{N}_{2}$ & $\mathrm{~g}$ & 0 & a & $191.502 \pm 0.025$ & $8.669 \pm 0.003$ & b \\
\hline C & $\mathrm{c}$ & 0 & a & $5.74 \pm 0.12$ & $1.050 \pm 0.020$ & q \\
\hline C & $\mathrm{g}$ & $716.67 \pm 0.44$ & $\mathrm{r}$ & $157.988 \pm 0.020$ & $6.535 \pm 0.006$ & $\mathrm{~b}$ \\
\hline $\mathrm{CO}$ & $\mathrm{g}$ & $-110.53 \pm 0.17$ & $\mathrm{~s}$ & $197.556 \pm 0.032$ & $8.673 \pm 0.008$ & b \\
\hline $\mathrm{CO}_{2}$ & $\mathrm{~g}$ & $-393.51 \pm 0.13$ & $\mathrm{t}$ & $213.677 \pm 0.040$ & $9.364 \pm 0.008$ & b \\
\hline
\end{tabular}

Tentative Set of Key Values for Thermodynamics-Part I, recalculated to calories*

\begin{tabular}{|c|c|c|c|c|}
\hline Substance & State & $\begin{array}{l}\Delta H_{\mathrm{f}, 298.15}^{\circ} \\
\mathrm{kcal} \mathrm{mol}^{-1}\end{array}$ & $\begin{array}{l}S_{298.15}^{\circ} \\
\text { cal } \mathrm{mol}^{-1} \mathrm{~K}^{-1}\end{array}$ & $\begin{array}{c}H^{\circ}{ }_{298.15}-H^{\circ}{ }_{0} \\
\mathrm{kcal} \mathrm{mol}^{-1}\end{array}$ \\
\hline $\mathrm{O}$ & $g$ & $59.553 \pm 0.024$ & $38.467 \pm 0.005$ & $1.608 \pm 0.001$ \\
\hline $\mathrm{O}_{2}$ & g & 0 & $49.005 \pm 0.008$ & $2.075 \pm 0.001$ \\
\hline $\mathrm{H}$ & $\mathrm{g}$ & $52.103 \pm 0.001 \dagger$ & $27.391 \pm 0.004$ & $1.481 \pm 0.001$ \\
\hline $\mathrm{H}^{+}$ & aq & 0 & 0 & - \\
\hline $\mathrm{H}_{2}$ & $\mathrm{~g}$ & 0 & $31.207 \pm 0.008$ & $2.024 \pm 0.001$ \\
\hline $\mathrm{H}_{2} \mathrm{O}$ & 1 & $-68.315 \pm 0.010$ & $16.718 \pm 0.019$ & - \\
\hline $\mathrm{H}_{2} \mathrm{O}$ & $\mathrm{g}$ & $-57.795 \pm 0.010$ & $45.106 \pm 0.010$ & $2.368 \pm 0.002$ \\
\hline
\end{tabular}




\begin{tabular}{|c|c|c|c|c|}
\hline ubstance & State & $\begin{array}{l}\Delta H_{\mathrm{f}, 298.15}^{\circ} \\
\mathrm{kcal} \mathrm{mol}^{-1}\end{array}$ & 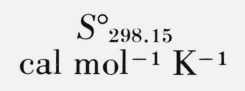 & $\begin{array}{l}H^{\circ}{ }_{298.15}-H^{\circ} \\
\mathrm{kcal} \mathrm{mol}^{-1}\end{array}$ \\
\hline $\mathrm{He}$ & $\mathrm{g}$ & 0 & $30.124 \pm 0.003$ & $1.481 \pm 0.001$ \\
\hline $\mathrm{Ne}$ & $\begin{array}{l}0 \\
g\end{array}$ & 0 & $34.946 \pm 0.004$ & $1.481 \pm 0.001$ \\
\hline Ar & $\mathrm{g}$ & 0 & $36.982 \pm 0.005$ & $1.481 \pm 0.001$ \\
\hline $\mathrm{Kr}$ & $\mathrm{g}$ & 0 & $39.190 \pm 0.005$ & $1.481 \pm 0.001$ \\
\hline $\mathrm{Xe}$ & g & 0 & $40.529 \pm 0.005$ & $1.481 \pm 0.001$ \\
\hline $\mathrm{Cl}$ & $\begin{array}{l}\text { o } \\
g\end{array}$ & $28.989 \pm 0.002$ & $39.454 \pm 0.005$ & $1.499 \pm 0.001$ \\
\hline $\mathrm{Cl}^{-}$ & $\mathrm{aq}$ & $-39.933 \pm 0.033$ & $13.57 \pm 0.14$ & - \\
\hline $\mathrm{Cl}_{2}$ & $\mathrm{~g}$ & 0 & $53.290 \pm 0.010$ & $2.194 \pm 0.002$ \\
\hline $\mathrm{HCl}$ & g & $-22.063 \pm 0.031$ & $44.643 \pm 0.008$ & $2.065 \pm 0.001$ \\
\hline $\mathrm{Br}$ & g & $26.730 \pm 0.029$ & $41.803 \pm 0.005$ & $1.481 \pm 0.001$ \\
\hline $\mathrm{Br}^{-}$ & aq & $-29.039 \pm 0.041$ & $19.91 \pm 0.31$ & - \\
\hline $\mathrm{Br}_{2}$ & 1 & 0 & $36.379 \pm 0.010$ & $5.860 \pm 0.031$ \\
\hline $\mathrm{Br}_{2}$ & g & $7.388 \pm 0.026$ & $58.640 \pm 0.013$ & $2.324 \pm 0.003$ \\
\hline $\mathrm{HBr}$ & g & $-8.695 \pm 0.041$ & $47.463 \pm 0.008$ & $2.067 \pm 0.001$ \\
\hline I & $\begin{array}{l}\text { g } \\
\mathrm{g}\end{array}$ & $25.517 \pm 0.010$ & $43.182 \pm 0.005$ & $1.481 \pm 0.001$ \\
\hline $\mathrm{I}^{-}$ & $\mathrm{aq}$ & $-13.60 \pm 0.20$ & $25.60 \pm 0.10$ & - \\
\hline $\mathrm{I}_{2}$ & $\mathrm{c}$ & 0 & $27.758 \pm 0.019$ & $3.154 \pm 0.010$ \\
\hline $\begin{array}{l}\mathrm{I}_{2}^{2} \\
\mathrm{I}_{2}\end{array}$ & g & $14.919 \pm 0.019$ & $62.277 \pm 0.015$ & $2.418 \pm 0.003$ \\
\hline HI & $\begin{array}{l}\text { o } \\
g\end{array}$ & $6.30 \pm 0.19$ & $49.350 \pm 0.010$ & $2.069 \pm 0.002$ \\
\hline $\mathrm{N}$ & $\mathrm{g}$ & $112.97 \pm 0.10$ & $36.613 \pm 0.005$ & $1.481 \pm 0.001$ \\
\hline $\mathrm{N}_{2}$ & $\begin{array}{l}\text { g } \\
\mathrm{g}\end{array}$ & 0 & $45.770 \pm 0.006$ & $2.072 \pm 0.001$ \\
\hline $\mathrm{C}^{2}$ & c & 0 & $1.372 \pm 0.029$ & $0.251 \pm 0.005$ \\
\hline $\mathrm{C}$ & $\mathrm{g}$ & $171.29 \pm 0.11$ & $37.760 \pm 0.005$ & $1.562 \pm 0.002$ \\
\hline $\mathrm{CO}$ & $\begin{array}{l}0 \\
\mathrm{~g}\end{array}$ & $-26.417 \pm 0.041$ & $47.217 \pm 0.008$ & $2.073 \pm 0.002$ \\
\hline $\mathrm{CO}_{2}$ & g & $-94.051 \pm 0.031$ & $51.070 \pm 0.010$ & $2.238 \pm 0.002$ \\
\hline
\end{tabular}

$\dagger$ Due to a calculation error these data were given in the original report as $218.002 \mathrm{~kJ} \mathrm{~mol}^{-1}\left(52.104 \mathrm{kcal}^{\mathrm{mol}}{ }^{-1}\right)$.

$* \mathrm{cal}=4.184 \mathrm{~J}$.

\section{Footnotes}

Defined reference state.

Calculated, by direct summation over the energy levels, at the Institute for High Temperatures (U.S.S.R.) and the National Bureau Standards (U.S.A.). The uncertainty given includes a contribution of $0.015 \%$ of the entropy due to the uncertainty in $R$.

c. Calculated using $D_{0}\left(\mathrm{O}_{2}\right)=41260 \pm 15 \mathrm{~cm}^{-1}$ from Brix and Herzberg (1954), assuming the products are $\mathrm{O}$ atoms in the ground state.

Calculated using $D_{0}\left(\mathrm{H}_{2}\right)=36118.3 \pm 1 \mathrm{~cm}^{-1}$ from Herzberg $(1970)$

Enthalpy of formation of $\mathrm{H}_{2} \mathrm{O}$ (1) from Rossini (1931, 1939), and King and Armstrong (1968).

f. Calculated from $S_{298.15}^{\circ} \mathrm{H}_{2} \mathrm{O}(\mathrm{g})$ with appropriate data for the entropies of expansion to saturation, correction to the real vapour and vaporization, from Keenan, Keyes, Hill, and Moore (1969). See also Giauque and Stout (1936).

g. Calculated from $\Delta H_{\mathrm{f}, 298.15}^{\circ} \mathrm{H}_{2} \mathrm{O}(1)$ using data from Keenan, Keyes, Hill, and Moore (1969).

1. Calculated using $D_{0}\left(\mathrm{Cl}_{2}\right)=19997 \pm 1 \mathrm{~cm}^{-1}$ based on Douglas, Møller, and Stoicheff (1963), LeRoy and Bernstein (1970), LeRoy (1970), and Sitterly (1970).

i. Enthalpy of formation of HCl(g) from Rossini (1932), Roth and Richter (1934), von Wartenberg and Hanisch (1932), Lacher, Kianpour, Oetting, and Park (1956), Faita, Longhi, and Mussini (1967), Cerquetti, Longhi, and Mussini (1968), and King and Armstrong (1970). Enthalpy of solution of HCl(g) from Gunn and Green (1963), Gunn (1964), Vanderzee and Nutter (1963), and Roth and Bertram (1937). Gibbs energy of solution from Bates and Kirschman (1919), Haase, Naas, and Thumm (1963), and Aston and (Gittler (1955). Entropy of $\mathrm{Cl}^{-}$(aq) from Bates and Bower (1954), and Greeley, Smith, Stoughton, and Lietzke (1960), on the Ag, AgCl, Cl- electrode. See also Cerquetti, Longhi, and Mussini (1968).

Calculated usin $D_{0}\left({ }^{79} \mathrm{Br}{ }^{81} \mathrm{Br}\right)=15893.1 \pm 2 \mathrm{~cm}^{-1}$ based on Horsley and Barrow (1967), and Sitterly (1970). LeRoy (1970) recalculated $D_{0}\left(\mathrm{Br}_{2}\right)$ from these data; his result is correct only if Horsley and Barrow missed many upper rotational levels of higher vibrational states in their interpretation of the absorption spectra.

Enthalpy of formation from Johnson and Sunner (1963), Johnson and Ambrose (1963), Sunner and Thorén (1964), Lacher, Casali, and 
Park (1956), and Howard and Skinner (1966). Enthalpy of solution of $\operatorname{HBr}(\dddot{m})$ from Vanderzee and Nutter (1963), Roth and Bertram (1937) and Thomsen (1884). The entropy of $\mathrm{Br}^{-}(\mathrm{aq})$ from measurements on the $\mathrm{Ag}, \mathrm{AgBr}_{\mathrm{g}} \mathrm{Br}^{-}$electrode by Hetzer, Robinson, and Bates (1962). Harned, Keston, and Donelson (1936), and Own and Foering (1936), and from differences in the entropy of solution for the salt pairs $\mathrm{KCl}-\mathrm{KBr}, \mathrm{CsCl}-\mathrm{CsBr}, \mathrm{AgCl}-\mathrm{AgBr}$, and NaCl-NaBr.2H.2.

1. From the specific heat and heat of vaporization measurements of Hildenbrand et al. (1958).

m. Calculated using $D_{0}\left(\mathrm{I}_{2}\right)=12440.9 \pm 1.1 \mathrm{~cm}^{-1}$ from LeRoy (1970), Brown (1931), LeRoy and Bernstein (1970), and Sitterly (1970).

n. Enthalpy of formation of $\mathrm{I}^{-}(\mathrm{aq})$ from Vorob'ev, Broier and Skuratov (1966), Wu, Birkey, and Hepler (1963), and Howard and Skinner (1966). Entropy of $\mathrm{I}^{-}$(aq) from measurements on the Ag, AgI, $I^{-}$electrode by Hetzer, Robinson, and Bates (1964), Kortüm and Häussermann (1965), and Feakins and Watson (1963), and from differences in the entropy of solution for the salt pairs KCl-KI, CsCl-CsI, AgCl-AgI, and $\mathrm{NaCl}-\mathrm{NaI} .2 \mathrm{H}_{2} \mathrm{O}$. Enthalpy of formation of $\mathrm{HI}(\mathrm{g})$ from Taylor and Crist (1941), Rittenberg and Urey (1934), Bright and Hagerty (1947), Murphy (1936), and Günther and Wekua (1931).

o. From the low-temperature specific heat data of Shirley and Giauque (1959), and vapour pressure data of Baxter, Hickey, and Holmes (1907), Baxter and Grose (1915), Gillespie and Fraser (1936), and Stern (1958).

p. Calculated from $D_{0}\left(\mathrm{~N}_{2}\right)=78715 \pm 50 \mathrm{~cm}^{-1}$ from Buttenbender and Herzberg (1935), Tanaka, Ogawa, and Jursa (1964), Tilford. Vanderslice and Wilkinson (1965), and Sitterly (1970). See the discussion in Gaydon (1968).

q. Graphite is selected as the standard reference state for carbon. However, there is considerable variation in the measured values of the low-temperature heat capacity and entropy, depending upon the source and nature of the graphite sample. See DeSorbo (1955), DeSorbo and Nichols (1958), DeSorbo and Tyler (1953). We have used the measurements of DeSorbo and Tyler on Acheson spectroscopic graphite, corresponding to the material used for the determination of the heat of formation of $\mathrm{CO}_{2}(\mathrm{~g})$.

r. Calculated using $D_{0}(\mathrm{CO})=89595 \pm 30 \mathrm{~cm}^{-1}$ from Douglas and Møller (1955), assuming the products are $\mathrm{C}\left({ }^{3} \mathrm{P}_{0}\right)$ and $\mathrm{O}\left({ }^{3} \mathrm{P}_{2}\right)$.

s. Heat of formation of $\mathrm{CO}(\mathrm{g})$ from combustion measurements of Rossini (1931, 1939), Awbery and Griffiths (1933), and Fenning and Cotton (1933), and equilibrium measurements by Smith (1946), Neumann and Köhler (1928), Peters and Mobius (1958), and Meyer and Scheffer (1938).

t. Heat of formation from Prosen and Rossini (1944), Hawtin, Lewis, Moul, and Phillips (1966), Dewey and Harper (1938), Jessup (1938), and Lewis, Frisch, and Margrave (1965). Much of the uncertainty relates to the difference in the various samples of graphite that have been studied.

\section{Bibliography}

Aston, J. G.: Gittler, F. L., J. Am. Chem. Soc. 77. 3173 (1955).

Awbery, J. H.; Griffiths, E., Proc. Roy. Soc. (London) A141, 1 (1933).

Bates, S. J.; Kirschman, H. D., J. Am. Chem. Soc. 41. 1991 (1919).

Bates, R. G.; Bower, V. E., J. Res. Nat. Bur. Stand. (U.S.), 53, 283 (1954).

Baxter, G. P.; Grose, M. R., J. Am. Chem. Soc. 37, 1061 (1915).

Baxter, G. P.; Hickey, C. H.: Holmes, W. C., J. Am. Chem. Soc. 29. 127 (1907).

Bright, N. F. H.; Hagerty, R. P.., Trans. Faraday Soc. 43, 697 (1947).

Brix, P.; Herzberg, G., Can. J. Phys. 32.110 (1954).

Brown, W. G., Phys. Rev. 38, 709 (1931).

Buttenbender, G.; Herzberg, G., Ann. Physik [5], 21, 577 (1935).

Cerquetti, A.; Longhi, P.; Mussini, T., J. Chem. Eng. Data 13, 458 (1968).

Commission on Atomic Weights of IUPAC, Pure Appl. Chem. 1970, 21,95 (1970).

DeSorbo, W., J. Chem. Phys. 23, 1970 (1955); J. Am. Chem. Soc. 7 7, 4713 (1955).

DeSorbo, W.: Nichols, G. F., J. Phys. Chem. Solids 6, 352 (1958).

DeSorbo, W.: Tyler, W. W.., J. Chem. Phys. 21, 1660 (1953).

Dewey, P. H.; Harper, D. R., J. Res. Nat. Bur. Stand. (U.S.), 2 1, 457 (1938).

Douglas, A. E.; Møller, C. K., Can. J. Phys. 33, 125 (1955).

Douglas, A. E.; Møller, C. K.; Stoicheff, B. P. Can. J. Phys. 41, 1174 (1963).

Faita, G.: Longhi, P.; Mussini, T., J. Electrochem. Soc. 114, 340 (1967).

Feakins, D.: Watson, P., J. Chem. Soc. 4686 (1963).

Fenning, R. W.; Cotton, F. T., Proc. Roy. Soc. (London) Al41, 17 (1933).

Gaydon. A. G., Dissociation Energies and Spectra of Diatomic Molecules (3d Ed. Chapman and Hall, London, 1968).

Giauque, W. F.; Stout, J. W., J. Am. Chem. Soc. 58, 1144 (1936). Gillespie, L. J.; Fraser, L. H. D., J. Am. Chem. Soc. 58, 2260 (1936). Greeley, R. S.; Smith, W. T.: Stoughton, R. W.; Lietzke, M. H., J. Phys. Chem. 64, 652 (1960).
Gunn, S. R., U.S. Atomic Energy Comm. Rept. UCRL 7992 (1964 Gunn. S. R.; Green, L. G.. J. Chem. Eng. Data 8. 180 (1963).

Günther, P.; Wekua, K., Z. Physik. Chem. A154, 193 (1931).

Haase, R.; Naas, H.: Thumm, H., Z. Physik. Chem. (Frankfurt) (N. F 37, 210 (1963).

Harned. H. S.: Keston, A. S.: Donelson, J. G., J. Am. Chem. Soc 58, 989 (1936).

Hawtin, P.:Lewis, J. B.; Moul, N.; Phillips, R. H., Trans. Roy. So (London) A261. 67 (1966).

Herzberg, G., J. Mol. Spectroscopy 33, 147 (1970).

Hetzer, H. B.: Robinson, R. A.: Bates, R. G., J. Phys. Chem. 6 1423 (1962).

Hetzer, H. B.; Robinson, R. A.; Bates, R. G., J. Phys. Chem. 6 1929 (1964).

Hildenbrand, D. L.; Kramer, W. R.: McDonald, R. A.; Stull, D. R J. Am. Chem. Soc. 80, 4129 (1958).

Horsley, T. A.: Barrow, R. F., Trans. Faraday Soc. 63, 32 (1967

Howard, P. B.: Skinner, H. A., J. Chem. Soc. 1536 (1966).

Jessup, R. S., J. Res. Nat. Bur. Stand. (U.S.), 21 , 475 (1938).

Johnson. W. H.; Ambrose, J. R., J. Res. Nat. Bur. Stand. (U.S. 67A (Phys. and Chem), 427 (1963).

Johnson, W. H.; Sunner, S., Acta Chem. Scand. 17, 1917 (1963

Keenan, J. H.; Keyes, F. G.; Hill, P. G.; Moore. J. G., Steam Table (John Wiley \& Sons, Inc., New York, 1969).

King. R. Armstrong, G. T., J. Res. Nat. Bur. Stand. (U.S.), 72 (Phys. and Chem.), No. 2, 113 (1968).

King, R.; Armstrong, G. T., J. Res. Nat. Bur. Stand. (U.S.), 74 (Phys. and Chem.), No. 6, 769 (1970).

Kortüm, G.; Häussermann, W. F., Ber. Bunsenges. Physik. Chen 69.594 (1965).

Lacher, J. R.; Casali, L.; Park, J. D., J. Phys. Chem. 60, 607 (1956

Lacher, J. R.: Kianpour, A.; Oetting, F.; Park, J. D., Trans. Farada Soc. 52, 1500 (1956).

LeRoy, R. T., Chem. Phys. Letters 5, 42 (1970).

LeRoy, R. T.: Bernstein, R. B., J. Chem. Phys. 52, 2678, 3869 (1970

Lewis, D. C.: Frisch, M. A.: Margrave, J. L., Carbon 2, 431 (1965

Meyer, G.; Scheffer, F. E. C., Rec. trav. chim. 5 7, 604 (1938).

Murphy, G. M.. J. Chem. Phys. 4. 344 (1936).

Neumann, B.: Kïhler, G., Z. Elektrochem. 34, 218 (1928). 
Owen, B. B.; Foering, L., J. Am. Chem. Soc. 58. 1575 (1936). Peters, H.; Mobius, H. H., Z. Physik. Chem. 209. 298 (1958). Prosen, E. J.; Rossini, F. D., J. Res. Nat. Bur. Stand. (U.S.), 33, 439 (1944).

Rittenberg, D.; Urey, H. C., J. Chem. Phys. 2 , 106 (1934).

Rossini, F. D., J. Res. Nat. Bur. Stand. (U.S.), 6, 1 and 36 (1931).

Rossini, F. D., J. Res. Nat. Bur. Stand. (U.S.), 9.679 (1932).

Rossini, F. D., J. Res. Nat. Bur. Stand. (U.S.), 22. 407 (1939).

Roth, W. A.; Bertram, A., Z. Elektrochem. 43,376 (1937).

Roth, W. A.; Richter, H., Z. Physik. Chem. Al 70. 123 (1934).

Shirley. D. A.: Giauque, W. F., J. Am. Chem. Soc. 81, 4778 (1959).

Sitterly, C. M., Nat. Bur. Stand. (U.S.), Washington, D.C. (Private commun.).

Smith, R. P., J. Am. Chem. Soc. 68. 1163 (1946).

Stern, T. H., Ph. D. Thesis. Univ. of Washington (1958).
Sunner, S.; Thorén, S., Acta Chem. Scand. 18, 1328 (1964).

Tanaka, Y.; Ogawa, M.; Jursa, A. S., J. Chem. Phys. 40, 3690 (1964).

Taylor, A. H.; Crist, K. H., J. Am. Chem. Soc. 63, 1377 (1941).

Thomsen, J., Thermochemische Untersuchungen (J. Barth, Leipzig, 1884).

Tilford, S. G.; Vanderslice, J. T.: Wilkinson, P. G., Astrophys. J. 142, 1203 (1965).

Vanderzee, C. E.; Nutter, J. D.. J. Phys. Chem. 67, 2521 (1963). Vorob'ev, A. F.: Broier, A. F.; Skuratov, S. M., Dokl. Akad. Nauk SSSR 1 73, 385 (1966).

von Wartenberg, H.; Hanisch, K., Z. Physik. Chem. A161, 413 (1932).

Wu, C.; Birkey, M. M.; Hepler, L. G., J. Phys. Chem. 67, 1202 (1963).

(Paper 75A2-651) 